4 Wilson JMG, Junger G. Principles and practice of screening for disease. Geneva: World Health Organization, 1968

5 Macdonald CE, Wicks AC, Playford RJ. Ten years' experience of screening patients with Barrett's oesophagus in a university teaching hospital. Gut 1997;41:303-7.

6 Caygill CPJ, Reed PI, Hill MJ, Watson A. An initial comparison of nine centres registering patients with the UK national Barrett's oesophagus registry (UKBOR). Eur J Cancer Prevention 1999;8:539-42.

7 Wright TA, Gray MR, Morris AI, Gilmore IT, Ellis A, Smart HL, et al. Cost effectiveness of detecting Barrett's cancer. Gut 1996;39:574-9.

8 Winters C, Spurling TJ, Chobanian SJ, Curtis DJ, Esposito RL, Hacker JF, et al. Barrett's esophagus: a prevalent, occult complication of gastroesophageal reflux disease. Gastroenterology 1987;92:118-24.

9 Johnsson F, Joelsson B, Gudmundsson K, Greiff L. Symptoms and endo- scopic findings in the diagnosis of gastrooesophageal reflux disease. Scand J Gastroenterol 1987;22:714-8.

10 Cameron AJ, Zinnmeister AR, Ballard DJ, Carey JA. Prevalence of columnar lined Barrett's esophagus: comparison of population based clinical and autopsy findings. Gastroenterology 1990;99:918-22.

11 Dent J, Bremner CG, Collen MJ, Hagg HRC, Spechler SJ, et al. Working party report to the world congress of gastroenterology, Sydney, 1990: Barrett's esophagus. J Gastroenterol Hepatol 1991;6:1-22

12 Smith AM, Maxwell-Armstrong CA, Welch NT, Scholefield JH. Surveillance for Barrett's oesophagus in the UK. Br J Surg 1999;86: 276-80.

13 Jankowski JA, Wright NA, Meltzer SJ, Triadafilopoulos G, Geboes K, Casson AG, et al. Molecular evolution of the metaplasia-dysplasiaadenocarcinoma sequence in the esophagus. Am J Path 1999;154:965-73. (Accepted 15 August 2000)

\title{
Maternal morbidity and mortality associated with interpregnancy interval: cross sectional study
}

\author{
Agustin Conde-Agudelo, José M Belizán
}

\begin{abstract}
Objective To study the impact of interpregnancy interval on maternal morbidity and mortality. Design Retrospective cross sectional study with data from the Perinatal Information System database of the Latin American Centre for Perinatology and Human Development, Montevideo, Uruguay. Setting Latin America and the Caribbean, 1985-97. Participants 456889 parous women delivering singleton infants.
\end{abstract}

Main outcome measures Crude and adjusted odds ratios of the effects of short and long interpregnancy intervals on maternal death, pre-eclampsia, eclampsia, gestational diabetes mellitus, third trimester bleeding, premature rupture of membranes, postpartum haemorrhage, puerperal endometritis, and anaemia. Results Short ( $<6$ months) and long ( $>59$ months) interpregnancy intervals were observed for $2.8 \%$ and $19.5 \%$ of women, respectively. After adjustment for major confounding factors, compared with those conceiving at 18 to 23 months after a previous birth, women with interpregnancy intervals of 5 months or less had higher risks for maternal death (odds ratio 2.54; $95 \%$ confidence interval 1.22 to 5.38), third trimester bleeding $(1.73 ; 1.42$ to 2.24$)$, premature rupture of membranes $(1.72 ; 1.53$ to 1.93$)$, puerperal endometritis $(1.33 ; 1.22$ to 1.45$)$, and anaemia (1.30; 1.18 to 1.43$)$. Compared with women with interpregnancy intervals of 18 to 23 months, women with interpregnancy intervals longer than 59 months had significantly increased risks of pre-eclampsia $(1.83 ; 1.72$ to 1.94$)$ and eclampsia $(1.80 ; 1.38$ to 2.32$)$. Conclusions Interpregnancy intervals less than 6 months and longer than 59 months are associated with an increased risk of adverse maternal outcomes.

\section{Introduction}

Both short and long interpregnancy intervals have been found to increase the risk of various adverse perinatal outcomes, such as low birth weight, preterm delivery, infants small for gestational age, stillbirth, and neonatal death..$^{1-5}$ The effect of interpregnancy interval on maternal morbidity and mortality has received less attention. In 1944, Eastman examined the effect of the interpregnancy interval, defined as "the interval between births," on some maternal outcomes in a cohort of 5158 parous women. ${ }^{6} \mathrm{He}$ found no association between interpregnancy interval and maternal anaemia, postpartum haemorrhage, puerperal fever, and maternal mortality. The risk of toxaemia, defined as pre-eclampsia and eclampsia with or without chronic hypertension, increased steadily with increasing interval between pregnancies. This investigation, however, did not control for confounding factors, and the number of women with short intervals was small. Since then, few studies have examined the association between interpregnancy interval and maternal outcomes. ${ }^{7-9}$ Two were case-control studies that looked only for association between interpregnancy interval and maternal mortality and provided apparently contradictory results ${ }^{79}$ : one showed an association whereas the other found no association. The other study evaluated the risk of anaemia according to intervals between pregnancies.

The Latin American and Caribbean Perinatal Information System database, which comprises information on maternal sociodemographic characteristics and outcomes of pregnancy, provides an opportunity to study the effects of interpregnancy interval on maternal morbidity and mortality.

\section{Participants and methods}

The Perinatal Information System database in Montevideo, Uruguay, was devised by the Latin American Centre for Perinatology and Human Development (CLAP) in $1983{ }^{10}$ Currently, this database is used for over half a million births each year. From 1985 to 1997 our database has recorded pregnancies of women who were born in Uruguay, Argentina, Peru, Colombia, Honduras, Paraguay, El Salvador, Chile, Bolivia, Costa Rica, Panama, Dominican Republic, Nicaragua, Brazil, Ecuador, Mexico, Bahamas, and Venezuela.

Only parous women delivering singleton infants and whose previous pregnancy ended in live birth or fetal death after 19 weeks' gestation were included in the study. A complete description of the database has been published elsewhere. ${ }^{11} 12$ From the first antenatal visit until discharge of both mother and neonate, the attending physicians or nurses collect data on demographic

\section{Latin American \\ Centre for \\ Perinatology and \\ Human \\ Development \\ (CLAP), Division of \\ Health Promotion \\ and Protection, Pan \\ American Health \\ Organisation, World \\ Health \\ Organisation, \\ Montevideo, \\ Uruguay \\ Agustin \\ Conde-Agudelo \\ perinatal researcher \\ José M Belizán \\ director \\ Correspondence to: A Conde-Agudelo condeagu@uniweb. net.co}

BMJ 2000;321:1255-9

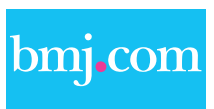

This article is part of the BMJ's randomised controlled trial of open peer review. Documentation relating to the editorial decision making process is available on the BMJ's website 
information, reproductive history, maternal characteristics, prenatal care, labour management, maternal complications during pregnancy, delivery, and the puerperium, and neonatal outcomes. Data are entered on to the database and cleaned on site by a data clerk. Queries on the database are checked immediately with the attendant physicians or nurses. Data are later sent to the Latin American Centre for Perinatology and Human Development, where a further data entry, quality control check, and validation is performed.

\section{Definitions}

Maternal age was defined as completed years at time of delivery. Mothers' education was categorised into none, elementary, secondary, and university. Marital status

Table 1 Distribution of sociodemographic and obstetric characteristics according to interpregnancy interval in cohort of 456889 Latin American and Caribbean women delivering singleton infants, 1985-97. Figures are percentage of women

\begin{tabular}{|c|c|c|c|c|c|c|}
\hline \multirow[b]{2}{*}{ Characteristic } & \multicolumn{6}{|c|}{ Interpregnancy interval (months) } \\
\hline & $\begin{array}{c}0-5 \\
(n=12704)\end{array}$ & $\begin{array}{c}6-11 \\
(n=63415)\end{array}$ & $\begin{array}{c}12-17 \\
(\mathrm{n}=67327)\end{array}$ & $\begin{array}{c}18-23 \\
(n=59372)\end{array}$ & $\begin{array}{c}24-59 \\
(n=164781)\end{array}$ & $\begin{array}{c}\geqslant 60 \\
(n=89290)\end{array}$ \\
\hline \multicolumn{7}{|c|}{ Maternal age (years): } \\
\hline$\leqslant 19$ & 20.0 & 18.0 & 14.7 & 9.7 & 4.5 & 0.6 \\
\hline $20-34$ & 72.3 & 74.2 & 75.9 & 79.3 & 81.6 & 68.9 \\
\hline$\geqslant 35$ & 7.7 & 7.8 & 9.4 & 11.0 & 13.9 & 30.5 \\
\hline \multicolumn{7}{|c|}{ No of previous deliveries: } \\
\hline 1 & 38.7 & 40.6 & 40.3 & 36.4 & 39.6 & 36.3 \\
\hline 2 & 26.1 & 23.0 & 23.9 & 23.9 & 24.7 & 29.4 \\
\hline$\geqslant 3$ & 35.2 & 36.4 & 35.8 & 39.7 & 35.7 & 34.3 \\
\hline \multicolumn{7}{|c|}{ History of miscarriage: } \\
\hline Yes & 40.7 & 27.4 & 23.3 & 22.7 & 24.3 & 27.1 \\
\hline No & 59.3 & 72.6 & 76.7 & 77.3 & 75.7 & 72.9 \\
\hline \multicolumn{7}{|c|}{ History of fetal death: } \\
\hline Yes & 5.7 & 5.5 & 4.4 & 4.2 & 3.3 & 2.7 \\
\hline No & 94.3 & 94.5 & 95.6 & 95.8 & 96.7 & 97.3 \\
\hline \multicolumn{7}{|c|}{ History of early neonatal death: } \\
\hline Yes & 4.4 & 4.2 & 3.2 & 3.8 & 2.9 & 2.8 \\
\hline No & 95.6 & 95.8 & 96.8 & 96.2 & 97.1 & 97.2 \\
\hline \multicolumn{7}{|c|}{ Previous caesarean delivery: } \\
\hline Yes & 11.7 & 16.2 & 15.5 & 16.9 & 15.8 & 13.5 \\
\hline No & 88.3 & 83.8 & 84.5 & 83.1 & 84.2 & 86.5 \\
\hline \multicolumn{7}{|c|}{ Mother living with infant's father: } \\
\hline Yes & 85.7 & 86.3 & 85.2 & 85.8 & 85.6 & 86.7 \\
\hline No & 14.3 & 13.7 & 14.8 & 14.2 & 14.4 & 13.3 \\
\hline \multicolumn{7}{|c|}{ Mother's education: } \\
\hline None & 3.7 & 3.7 & 4.6 & 4.5 & 4.4 & 3.6 \\
\hline Elementary & 51.9 & 54.5 & 54.6 & 54.6 & 51.2 & 52.0 \\
\hline Secondary & 40.0 & 37.1 & 35.5 & 36.5 & 39.2 & 40.2 \\
\hline University & 4.4 & 4.7 & 5.3 & 4.4 & 5.2 & 4.2 \\
\hline \multicolumn{7}{|c|}{ Gestation at start of prenatal care (weeks): } \\
\hline $1-13$ & 17.6 & 22.0 & 24.8 & 25.5 & 31.0 & 34.5 \\
\hline $14-26$ & 44.2 & 45.7 & 43.7 & 44.6 & 43.3 & 44.6 \\
\hline$\geqslant 27$ & 38.2 & 32.3 & 31.5 & 29.9 & 25.7 & 20.9 \\
\hline \multicolumn{7}{|c|}{ No of prenatal visits: } \\
\hline 0 & 27.7 & 23.2 & 21.7 & 21.2 & 17.3 & 12.8 \\
\hline $1-4$ & 37.2 & 39.2 & 37.0 & 36.5 & 32.1 & 27.3 \\
\hline$\geqslant 5$ & 35.1 & 37.6 & 41.3 & 42.3 & 50.6 & 59.9 \\
\hline \multicolumn{7}{|c|}{ Cigarette smoking: } \\
\hline Yes & 12.4 & 11.5 & 11.3 & 11.7 & 11.8 & 12.6 \\
\hline No & 87.6 & 88.5 & 88.7 & 88.3 & 88.2 & 87.4 \\
\hline \multicolumn{7}{|c|}{ Body mass index $\left(\mathrm{kg} / \mathrm{m}^{2}\right)$ before pregnancy: } \\
\hline$<19.8$ & 16.2 & 16.4 & 16.9 & 16.8 & 16.5 & 10.2 \\
\hline $19.8-26.0$ & 64.8 & 61.8 & 61.9 & 63.8 & 61.5 & 61.0 \\
\hline $26.1-29.0$ & 10.9 & 13.4 & 11.5 & 9.5 & 11.1 & 14.2 \\
\hline$>29.0$ & 8.1 & 8.4 & 9.7 & 9.9 & 10.9 & 14.6 \\
\hline \multicolumn{7}{|c|}{ History of chronic hypertension: } \\
\hline Yes & 0.9 & 1.2 & 1.2 & 1.6 & 1.6 & 3.3 \\
\hline No & 99.1 & 98.8 & 98.8 & 98.4 & 98.4 & 96.7 \\
\hline
\end{tabular}

was dichotomised between those who did and did not live with their infant's father. Maternal height and weight before pregnancy were recorded by recall at the woman's first antenatal visit in centimetres and kilograms, respectively. The body mass index (weight $(\mathrm{kg}) /$ (height $(\mathrm{m})^{2}$ before pregnancy) was categorised as underweight (body mass index <19.8); normal (19.8-26.0); overweight (26.1-29.0); and obese $(>29.0) \cdot{ }^{13}$ Information on cigarette smoking was also recorded at the first antenatal visit and categorised into smoker and non-smoker.

Gestational age was estimated from the date of last menstrual period and amended by means of ultrasonography in a quarter of women. Interpregnancy interval was defined as the time elapsed between the woman's last delivery and the date of the last menstrual period for the index pregnancy. Intervals were computed in weeks and then converted to months. Interpregnancy intervals were categorised as $\leqslant 5,6-11$, $12-17,18-23,24-59$, and $\geqslant 60$ months.

Adverse maternal outcomes were classified according to ICD-10 (international classification of diseases, 10th revision). Pre-eclampsia and eclampsia were codes O14 and O15, respectively. Third trimester bleeding included placenta praevia with haemorrhage (code O44.1) and placental abruption (code O45). Anaemia, premature rupture of membranes, gestational diabetes mellitus, postpartum haemorrhage, and puerperal endometritis were codes O99.0, O42, O24.4, O72, and O85, respectively.

Maternal death was defined as the death of a woman while she was pregnant or within 42 days after delivery from any cause related to or aggravated by the pregnancy or its management but not from accidental or incidental causes.

\section{Analysis}

Rates of adverse maternal outcomes were calculated for each interpregnancy interval. Estimates of crude odds ratios with 95\% confidence intervals were computed as measures of association between each interpregnancy interval and adverse maternal outcome considered. The interval 18-23 months was used as the reference category because this was the interval during which maternal death was least likely to occur. Adjusted odds ratios were derived through logistic regression models. Multiple logistic regression analysis was used to evaluate the association between interpregnancy interval and adverse maternal outcomes. The estimates were adjusted for 16 major confounding factors.

All analyses were done with the SPSS 8.0 program package (SPSS, Chicago).

\section{Results}

A total of 520689 parous women who delivered singleton infants between 1985 and 1997 were recorded on our database. The final study population included 456889 women whose records contained complete data on interpregnancy interval and adverse maternal outcomes. There were no significant differences between the women excluded because of incomplete data and those with complete data with regard to maternal age, parity, education, and marital status. 
The median interpregnancy interval was 27 months. Short ( $<6$ months) and long ( $>59$ months) intervals between pregnancies were observed for $2.8 \%$ and $19.5 \%$ of women, respectively. Almost a third of the women had an interpregnancy interval of less than 18 months.

Table 1 shows the characteristics of the mothers at the index pregnancy according to interpregnancy interval. Younger maternal age, history of miscarriage, fetal death and early neonatal death, lower rate of previous caesarean delivery, later start of prenatal care, lower number of prenatal visits, and lower body mass index before pregnancy were associated with short intervals between pregnancies. Conversely, women with a long interpregnancy interval were more likely to be older, with greater body mass index before pregnancy, and with a history of chronic hypertension. Start of prenatal care and number of prenatal visits correlated with interpregnancy interval: the shorter the interval, the later care started and the lower the number of prenatal visits. There were no obvious differences among the interpregnancy interval groups with regard to number of previous deliveries, mother's education, marital status, and cigarette smoking during pregnancy.

Women with short interpregnancy intervals had the highest rates of third trimester bleeding, premature rupture of membranes, puerperal endometritis, anaemia, and maternal death (table 2). There were 220 maternal deaths in the study population The rates of pre-eclampsia, eclampsia, and gestational diabetes mellitus were highest among women with intervals longer than 59 months. A slight increase in the rates of third trimester bleeding and maternal death was also seen in women with this interpregnancy interval.

Table 3 shows the results of multiple logistic regression analysis of the relation of interpregnancy intervals to adverse maternal outcomes. Compared with mothers with interpregnancy intervals of 18 to 23 months, mothers with intervals shorter than 6 months had about a $70 \%$ increased risk of third trimester bleeding and premature rupture of membranes and a $30 \%$ increased risk of anaemia and puerperal endometritis. Moreover, a short interval between pregnancies was associated with a significantly greater risk of maternal death (adjusted odds ratio 2.54; $95 \%$ confidence interval 1.22 to 5.38). When interpregnancy intervals were dichotomised to shorter than 6 months
Table 2 Rates of adverse maternal outcomes according to interpregnancy interval in cohort of 456889 Latin American and Caribbean parous women delivering singleton infants, 1985-97. Figures are percentage of women

\begin{tabular}{|c|c|c|c|c|c|c|c|}
\hline \multirow[b]{2}{*}{ Outcome } & \multicolumn{7}{|c|}{ Interpregnancy interval (months) } \\
\hline & $0-5$ & $6-11$ & $12-17$ & $18-23$ & $24-59$ & $\geqslant 60$ & Overall \\
\hline Pre-eclampsia & 3.4 & 3.2 & 3.3 & 3.4 & 4.2 & 6.6 & 4.3 \\
\hline Eclampsia & 0.12 & 0.10 & 0.12 & 0.11 & 0.10 & 0.20 & 0.13 \\
\hline Third trimester bleeding & 1.9 & 1.2 & 1.1 & 1.1 & 1.1 & 1.5 & 1.2 \\
\hline Premature rupture of membranes & 9.8 & 5.9 & 5.6 & 5.6 & 6.6 & 6.5 & 6.7 \\
\hline Postpartum haemorrhage & 5.1 & 5.6 & 5.4 & 5.5 & 5.3 & 5.3 & 5.3 \\
\hline Puerperal endometritis & 5.1 & 4.2 & 4.4 & 4.0 & 3.9 & 4.3 & 4.1 \\
\hline Gestational diabetes mellitus & 1.4 & 1.2 & 1.0 & 1.4 & 1.4 & 2.4 & 1.6 \\
\hline Anaemia & 7.9 & 6.5 & 6.3 & 6.1 & 6.2 & 6.2 & 6.3 \\
\hline Maternal death* & 9.5 & 4.4 & 4.2 & 3.7 & 4.9 & 5.5 & 4.8 \\
\hline
\end{tabular}

*Rate/10 000 women.

versus 6 months or more, women with short intervals between pregnancies were significantly more likely to die than women conceiving at or after 6 months (2.04; 1.13 to 3.78). On the other hand, women with interpregnancy intervals of 60 months or more were 1.8 times more likely than women with intervals of 18 to 23 months to develop pre-eclampsia and eclampsia. We found no significant differences in the effect of interpregnancy interval on gestational diabetes mellitus, and no relation between the interval and the risk of postpartum haemorrhage.

\section{Discussion}

Our results indicate that women with short intervals ( $<6$ months) between pregnancies are at increased risk of maternal death, third trimester bleeding, premature rupture of membranes, puerperal endometritis, and anaemia. Likewise, long intervals (>59 months) were associated with higher risks of pre-eclampsia and eclampsia. Our findings are supported by our large sample size, which confers sufficient power to evaluate the relation between interpregnancy interval and adverse maternal outcomes and by our ability to control for the influence of many possible confounding factors.

Few studies have examined the effect of interpregnancy interval on maternal outcomes. An earlier study from the United States did not find any effect of interpregnancy interval on maternal anaemia, puerperal fever, postpartum haemorrhage, and maternal mor-

Table 3 Adjusted relative risk (95\% confidence interval) for adverse maternal outcomes according to interpregnancy interval in cohort of 456889 Latin American and Caribbean parous women delivering singleton infants, 1985-97*

\begin{tabular}{|c|c|c|c|c|c|c|}
\hline \multirow[b]{2}{*}{ Outcome } & \multicolumn{6}{|c|}{ Interpregnancy interval (months) } \\
\hline & $0-5$ & $6-11$ & $12-17$ & $18-23 \dagger$ & $24-59$ & $\geqslant 60$ \\
\hline Pre-eclampsia & 1.00 (0.93 to 1.07$)$ & 0.98 (0.88 to 1.08$)$ & 1.03 (0.93 to 1.14$)$ & 1.0 & $1.06(0.96$ to 1.18$)$ & $1.83(1.72$ to 1.94$)$ \\
\hline Eclampsia & $1.12(0.63$ to 2.29$)$ & 1.04 (0.68 to 1.43$)$ & 0.97 (0.78 to 1.19$)$ & 1.0 & 1.22 (0.84 to 2.33) & $1.80(1.38$ to 2.32$)$ \\
\hline Third trimester bleeding & $1.73(1.42$ to 2.24$)$ & $1.03(0.91$ to 1.16$)$ & 1.01 (0.88 to 1.14$)$ & 1.0 & $1.04(0.96$ to 1.14$)$ & $1.12(1.00$ to 1.24$)$ \\
\hline Premature rupture of membranes & 1.72 (1.53 to 1.93$)$ & 1.04 (0.96 to 1.12$)$ & 1.02 (0.93 to 1.12$)$ & 1.0 & 1.08 (0.98 to 1.19) & $1.03(0.93$ to 1.14$)$ \\
\hline Postpartum haemorrhage & 0.94 (0.76 to 1.13$)$ & $0.96(0.87$ to 1.06$)$ & 0.98 (0.90 to 1.06$)$ & 1.0 & 0.95 (0.86 to 1.04$)$ & $0.91(0.78$ to 1.04$)$ \\
\hline Puerperal endometritis & 1.33 (1.22 to 1.45$)$ & $1.04(0.94$ to 1.14$)$ & 1.08 (1.00 to 1.17$)$ & 1.0 & 0.99 (0.94 to 1.04) & $1.04(0.94$ to 1.15$)$ \\
\hline Gestational diabetes mellitus & $1.02(0.71$ to 1.35$)$ & 0.95 (0.84 to 106$)$ & 0.91 (0.69 to 1.14$)$ & 1.0 & 1.03 (0.83 to 1.23$)$ & $1.25(0.88$ to 1.63$)$ \\
\hline Anaemia & 1.30 (1.18 to 1.43$)$ & 1.03 (0.95 to 1.12$)$ & 1.02 (0.96 to 1.09$)$ & 1.0 & $1.04(0.99$ to 1.10$)$ & $1.01(0.97$ to 1.05$)$ \\
\hline Maternal death & 2.54 (1.22 to 5.38$)$ & 1.11 (0.53 to 2.28$)$ & 1.03 (0.56 to 2.22$)$ & 1.0 & 1.14 (0.63 to 2.41$)$ & $1.07(0.71$ to 2.71$)$ \\
\hline
\end{tabular}

${ }^{*}$ Adjusted for maternal age, No of previous deliveries, history of miscarriage, stillbirth and early neonatal death, previous caesarean delivery, marital status,

education, cigarette smoking, body mass index before pregnancy, trimester during which prenatal care was started, No of prenatal care visits, geographic area,

hospital type, and year of delivery. Pre-eclampsia, eclampsia, gestational diabetes mellitus, and third trimester bleeding were also adjusted for history of chronic hypertension.

†Reference group. 
tality, although the number of maternal deaths ${ }^{16}$ was small. ${ }^{6}$ An increased risk of maternal mortality among Hindu women with intervals between pregnancies of less than 24 months compared with women with interpregnancy intervals of 24 or more months (relative risk 2.5; $95 \%$ confidence interval 1.5 to 4.3 ) has been reported in a case-control study of 252 maternal deaths matched to 252 survivors by age, parity, and booking status. ${ }^{7}$ As a strong association between maternal death and both age and parity was also reported, however, the results of this study are difficult to interpret. A recent nested case-control study from Bangladesh evaluated risk factors for 390 maternal deaths. ${ }^{9}$ It found that interpregnancy intervals shorter than 9 months did not increase the risk of maternal death (adjusted odds ratio 1.29; 95\% confidence interval 0.77 to 2.14) compared with interpregnancy intervals of 15 to 26 months, after adjustment for maternal age, area of residence, maternal education, religion, and year of birth. ${ }^{9}$ None the less, women dying within 90 days after the end of pregnancy and with external causes of death such as induced abortion or suicide were included in the study, which may have hidden the existence of an association. In addition, gestational age was unknown for $44 \%$ of pregnancies, thereby biasing calculation of interpregnancy interval.

\section{Short interpregnancy intervals and adverse maternal outcomes}

Our finding of higher rates of anaemia among women with short interpregnancy intervals agrees with findings of previous studies. ${ }^{814}$ With regard to third trimester bleeding a recent population based study found a greater risk of uteroplacental bleeding disorders among young multiparous women. ${ }^{15}$ The authors attributed it to a higher incidence of short intervals between pregnancies in these women. Our results confirmed such an association.

The reasons for the association between a short interval between pregnancies and adverse maternal outcomes are unclear. Most of our findings might be explained by the maternal depletion hypothesis, which suggests that short intervals do not allow to the mother to recover from the physiological stresses imposed by the previous pregnancy. ${ }^{16-18}$ This results in depletion of maternal nutrient stores and anaemia, which have been found to play a part in the pathogenesis of premature rupture of membranes and puerperal endometritis. ${ }^{19} 20$ With regard to the increased risk of third trimester bleeding, we postulate that a short interval between pregnancies might interfere in normal processes of remodelling of endometrial blood vessels after delivery, with subsequent uteroplacental underperfusion, ${ }^{21}$ thereby increasing the likelihood for placental abruption and placenta praevia. All the previously mentioned conditions may contribute to the increased risk of maternal death among women with short interpregnancy intervals found in our study. Other alternative explanations for the relation between short interpregnancy intervals and adverse maternal outcomes might be postpartum stress levels, socioeconomic factors other than marital status and education, unstable lifestyles, occupation, community variables (for example, crime, drug misuse, housing), failure to use healthcare services or inadequate use of such services, and other behavioural or psychological determinants.

\section{Long interpregnancy intervals and adverse}

\section{maternal outcomes}

Results from our study corroborate the finding from an earlier report that showed that women with long intervals between pregnancies are at increased risk of pre-eclampsia. Eastman reported that compared with mothers with interpregnancy intervals of 12 to 23 months mothers with intervals longer than 48 months had a significantly greater risk of toxaemia (relative risk $1.3 ; 95 \%$ confidence interval 1.1 to 1.5$){ }^{6}$ Interestingly, the rate of pre-eclampsia among nulliparous women recorded in our database $(n=325146)$ was similar to that of parous women who conceived five or more years after a previous birth $(6.5 \%$ versus $6.6 \%$, respectively). It would seem that parous women with long interpregnancy intervals behave as nulliparous women with regard to the risk of pre-eclampsia as if the "protective" effect for pre-eclampsia acquired by a woman through a previous birth is lost after a long interval. Certain variables that may confound or modify the relations between interpregnancy interval and pre-eclampsia, however, such as change in paternity, ${ }^{22}$ were not available to us for analysis. Regardless of what causes this association, women who become pregnant a long time after the previous pregnancy are at higher risk of pre-eclampsia and eclampsia and need to be carefully monitored during antenatal care. On the other hand, after adjustment for confounders, the effect of long interpregnancy intervals on gestational diabetes mellitus disappeared. Thus, the increased rate of gestational diabetes mellitus associated with intervals greater than 59 months could be mediated through older maternal age and higher body mass index before pregnancy.

\section{Methodological considerations}

Several limitations and potential biases of this study must be considered. Firstly, socioeconomic factors are potential confounders for the relation between interpregnancy interval and adverse maternal outcomes. In the present study, we accounted for only two of these factors (maternal education and cohabitation of parents), but we were unable to evaluate the relation to other socioeconomic factors such as family income and race because these data were not available from the database. Secondly, our study was not population based. Rather, it was based at several different hospitals spread across Latin American and the Caribbean. In general, less than 2\% of all Latin American and Caribbean births are represented by our database. It is unlikely, however, that the effect of interpregnancy interval on adverse maternal outcomes had been confounded by this matter as the results were similar among the countries considered in our study. Thirdly, the accuracy of specific diagnoses registered on this large database has not been extensively investigated and only local medical records have been verified. ${ }^{23}$ As such, data from the database are limited to a certain extent. Fourthly, despite adjustment for several variables, there is still potential for confounding by other unknown factors. Fifthly, inaccuracy of gestational age estimated from the date of last menstrual period is a well recognised problem in epidemiological research on interpregnancy intervals. When we replicated the entire analyses using gestational age estimated from physical and neurological assessments of the newborn instead of that based on last menstrual period, the 
results were essentially unchanged. Finally, it should be emphasised that our study was carried out in developing countries and its findings may therefore not be applicable to other populations.

The risks for maternal perinatal morbidity and mortality associated with short interpregnancy intervals underscore the importance of birth spacing by using the available methods of family planning, particularly after a birth, to promote safe motherhood and achieve better pregnancy outcomes. In addition, women should be advised of the potential harm to them and their infants of short and long intervals between pregnancies.

We thank Mr Roberto Porro and Dr Felipe Santana for the preparation of the database. We also thank health workers and health related workers in many settings of the Latin American and Caribbean region for their efforts to collect and send Perinatal Information System data to the Latin American Centre for Perinatology and Human Development.

Contributors: AC-A originated and designed the study, analysed the data, and was mainly responsible for writing the paper; he will act as guarantor of the paper. JMB participated in the discussion of the study hypothesis and study design, contributed to the analyses, and helped to write the paper.

Funding: Division of Health Promotion and Protection, Pan American Health Organisation.

Competing interests: None declared.

1 Rawlings JS, Rawlings VB, Read JA. Prevalence of low birth weight and preterm delivery in relation to the interval between pregnancies among white and black women. N Engl J Med 1995;332:69-74.

2 Klerman LV, Cliver SP, Goldenberg RL. The impact of short interpregnancy intervals on pregnancy outcomes in a low-income population. Am J Public Health 1998;88:1182-5.

3 Khoshnood B, Lee KS, Wall S, Hsieh HL, Mittendorf R. Short interpregnancy intervals and the risk of adverse birth outcomes among five racial ethnic groups in the United States. Am J Epidemiol 1998;148:798-805.

4 Zhu BP, Rolfs RT, Nangle BE, Horan JM. Effect of the interval between pregnancies on perinatal outcomes. N Engl J Med 1999;340:589-94

5 Shults RA, Arndt V, Olshan AF, Martin CF, Royce RA. Effects of short interpregnancy intervals on small-for-gestational age and preterm births. Epidemiology 1999;10:250-4.

6 Eastman NJ. The effect of the interval between births on maternal and fetal outlook. Am J Obstet Gynecol 1944;47:445-66.

7 Anandalakshmy PN, Talwar PB, Buckshee K, Hingorani V. Demographic, socio-economic and medical factors affecting maternal mortality-an Indian experience. J Fam Welfare 1993;39:1-4

8 Lazovic N, Pocekovac P. The importance of time intervals between childbirth and anemia in pregnancy. Srp Arh Celok Lek 1996;124:307-10. (In Serbo-Croatian.)

9 Ronsmans C, Campbell O. Short birth intervals don't kill women: evidence from Matlab, Bangladesh. Stud Fam Plann 1998;29:282-90.

10 Schwarcz R, Diaz AG, Fescina R, Diaz JL, Martell M, Simini F. The perinatal information system. I: The simplified perinatal clinical record. J Perinat Med 1987;15(suppl 1):9.

\section{What is already known on this topic?}

Both short and long intervals between pregnancies are associated with adverse perinatal outcomes

Conflicting data exist on the impact of interpregnancy interval on maternal morbidity and mortality

\section{What does this study add?}

Women with interpregnancy intervals shorter than 6 months are at increased risk of maternal death, third trimester bleeding, premature rupture of membranes, puerperal endometritis, and anaemia

Women with interpregnancy intervals longer than 59 months are at increased risk of pre-eclampsia and eclampsia

As the research was carried out in developing countries the results may not apply to other populations

11 Diaz-Rosello JL. Health services research, outcomes, and perinatal information systems. Curr Opin Pediatr 1998;10:117-22.

12 Simini F. Perinatal information system (SIP): a clinical database in Latin America and the Caribbean. Lancet 1999;354:75.

13 Institute of Medicine, National Academy of Sciences. Nutrition during pregnancy and lactation. An implementation guide. Washington, DC: National Academy Press, 1992.

14 Mahfouz AA, el-Said MM, Alakija W, Badawi IA, al-Erian RA, Moneim MA. Anemia among pregnant women in the Asir region, Saudi Arabia: an epidemiologic study. Southeast Asian J Trop Med Public Health 1994;25:84-7.

15 Ananth CV, Wilcox AJ, Savitz DA, Bowes WA Jr, Luther ER. Effect of maternal age and parity on the risk of uteroplacental bleeding disorders in pregnancy. Obstet Gynecol 1996;88:511-6.

16 Miller JE. Birth intervals and perinatal health: an investigation of three hypotheses. Fam Plann Perspect 1991;23:62-70.

17 Winkvist A, Rasmussen KM, Habicht JP. A new definition of maternal depletion syndrome. Am J Public Health 1992;82:691-4.

18 Khan KS, Chien PF, Khan NB. Nutritional stress of reproduction. Acta Obstet Gynecol Scand 1998;77:395-401.

19 Alger LS, Pupkin MJ. Etiology of preterm premature rupture of the membranes. Clin Obstet Gynecol 1986;29:758-70.

20 Libombo A, Folgosa E, Bergstrom S. Risk factors in puerperal endometritis-myometritis. An incident case-referent study. Gynecol Obstet Invest 1994:38:198-205.

21 Naeye RL. Maternal age, obstetric complications, and the outcome of pregnancy. Obstet Gynecol 1983;61:210-6.

22 Trupin LS, Simon LP, Eskenazi B. Change in paternity: a risk factor for preeclampsia in multiparas. Epidemiology 1996;7:240-4.

23 Cobo E. Aplicación de un modelo de historia clínica perinatal. Rev Col Obstet Ginecol 1985;36:79-94.

(Accepted 11 September 2000)

\title{
The timing of the "fertile window" in the menstrual cycle: day specific estimates from a prospective study
}

\author{
Allen J Wilcox, David Dunson, Donna Day Baird
}

\author{
Abstract \\ Objectives To provide specific estimates of the likely \\ occurrence of the six fertile days (the "fertile window") \\ during the menstrual cycle. \\ Design Prospective cohort study. \\ Participants 221 healthy women who were planning \\ a pregnancy. \\ Main outcome measures The timing of ovulation in \\ 696 menstrual cycles, estimated using urinary \\ metabolites of oestrogen and progesterone. \\ Results The fertile window occurred during a broad \\ range of days in the menstrual cycle. On every day
}

between days 6 and 21, women had at minimum a $10 \%$ probability of being in their fertile window. Women cannot predict a sporadic late ovulation; $4-6 \%$ of women whose cycles had not yet resumed were potentially fertile in the fifth week of their cycle. Conclusions In only about $30 \%$ of women is the fertile window entirely within the days of the menstrual cycle identified by clinical guidelines-that is, between days 10 and 17. Most women reach their fertile window earlier and others much later. Women should be advised that the timing of their fertile window can be highly unpredictable, even if their cycles are usually regular. 